Fetal Diagnosis and Therapy
Fetal Diagn Ther 2017;41:283-292

DOI: $10.1159 / 000448946$
Received: December 1, 2015

Accepted after revision: August 5, 2016

Published online: November 4, 2016

\title{
Quality Predictors of Abdominal Fetal Electrocardiography Recording in Antenatal Ambulatory and Bedside Settings
}

\author{
Evelyn A. Huhn ${ }^{a}$ Maren I. Müller ${ }^{b}$ Andrea H. Meyer ${ }^{b}$ \\ Gwendolin Manegold-Brauer ${ }^{a}$ Wolfgang Holzgreve ${ }^{c}$ Irene Hoeslia \\ Frank H. Wilhelm ${ }^{d}$ \\ ${ }^{a}$ Department of Obstetrics and Gynecology, University Hospital Basel, and bepartment of Clinical Psychology

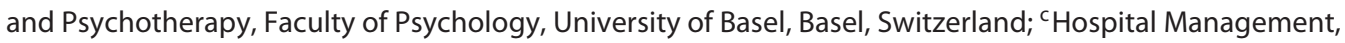

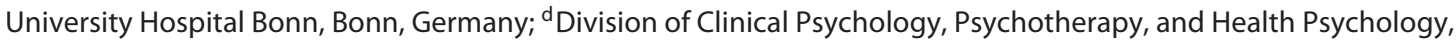 \\ Department of Psychology, University of Salzburg, Salzburg, Austria
}

\section{Key Words}

Fetal electrocardiography · Fetal heart rate - Ambulatory monitoring $\cdot$ Heart rate variability · Monica AN24 ${ }^{\mathrm{TM}}$.

Abdominal electrocardiography

\begin{abstract}
Background: Fetal electrocardiography using an abdominal monitor (Monica AN24 ${ }^{\mathrm{TM}}$ ) could increase the diagnostic use of fetal heart rate (fHR) variability measurements. However, signal quality may depend on factors such as maternal physical activity, posture, and bedside versus ambulatory setting. Methods: Sixty-three healthy women wore the monitor at home and 42 women during a hospital stay. All women underwent a posture experiment, and all home and 13 hospital participants wore the monitor during daytime and nighttime. The success rate (SR) of fHR detection was analyzed in relation to maternal physical activity, posture, daytime versus nighttime, and other maternal and fetal predictors. Results: Ambulatorily, the SR was $86.8 \%$ for nighttime and $40.2 \%$ for daytime. The low daytime SR was largely due to effects of maternal physical activity and posture. The in-hos-
\end{abstract}

\section{KARGER}

(C) 2016 S. Karger AG, Basel pital SR was lower during nighttime (71.1\%) and similar during daytime (43.3\%). SR was related to gestational age, but not affected by pre-pregnancy and current body mass index or fetal growth restriction. Conclusions: The success of beatto-beat fHR detection strongly depends on the home/hospital setting and predictors such as time of recording, activity levels, and maternal posture. Its clinical utility may be limited in periods of unsupervised recording with physical activity or posture shifts.

(c) 2016 S. Karger AG, Basel

\section{Introduction}

Fetal heart rate (fHR) monitoring is an important method of fetal surveillance in high-risk pregnancies. The first commercially available noninvasive abdominal fetal electrocardiography (fECG) monitor, the Monica AN24 ${ }^{\mathrm{TM}}$ (Monica Healthcare Ltd., Nottingham, UK), can obtain beat-to-beat fHR and fECG morphology signals with an effective sample rate of $2.1 \mathrm{kHz}$ over extended periods. Indications for continuous antenatal fECG mon-

Evelyn A. Huhn

Department of Obstetrics and Gynecology University Hospital Basel

Spitalstrasse 21, CH-4031 Basel (Switzerland)

E-Mail evelyn.huhn@usb.ch 
itoring are high-risk pregnancies, including fetal growth restriction (FGR), subsequent pregnancy after unexplained intrauterine death, or fetal cardiac arrhythmias. The Monica AN24 ${ }^{\mathrm{TM}}$ can be combined with a home care mobile phone application, allowing home surveillance of the fetus as an alternative to a costly hospital stay.

The clinical viability of an fECG device depends on accurate fHR detection. Noninvasive fECG has shown good accuracy of fECG monitoring prenatally when directly compared to gold standard Doppler ultrasound techniques such as cardiotocography (CTG) (see table 1 [113] for a comparison between fECG and CTG). The utility of the fHR signal hinges particularly on low rates of signal dropout to allow for reliable scoring of clinically important fHR variability (fHRV) measures. However, detection of the typically weak fECG from maternal abdominal surface electrodes is difficult due to interference from maternal ECG and can be compromised due to maternal muscle activity (particularly from the abdomen) and other factors. Thus, it is important to delineate under which exact circumstances the fHR signal is available with fECG monitoring.

The aim of the current study was to test the Monica AN24 ${ }^{\mathrm{TM}}$ device in its utility for antenatal assessment in various populations and settings, including ambulatory and heterogeneous hospital bedside settings. We specifically evaluated variables likely contributing to signal dropout, such as maternal body mass index (BMI), physical activity and posture, daytime versus nighttime recording, gestational age, and fetal presentation and condition.

Table 1. Advantages and disadvantages of abdominal fECG (with emphasis on Monica AN24 ${ }^{\mathrm{TM}}$ ) versus standard CTG

\begin{tabular}{|c|c|c|}
\hline Abdominal fECG & CTG & Validating fECG study \\
\hline \multicolumn{3}{|l|}{ Advantages } \\
\hline $\begin{array}{l}\text { Beat-to-beat fHRV with high temporal } \\
\text { resolution of } \mathrm{R} \text { wave detection }\end{array}$ & No determination of single R-R intervals & $\begin{array}{l}\text { Information by Monica Healthcare, } \\
\text { Nottingham, UK }\end{array}$ \\
\hline $\begin{array}{l}\text { Good SR in long-term recordings and } \\
\text { overnight; no belt/transducer adjustments }\end{array}$ & $\begin{array}{l}\text { Used for short durations at regular intervals; } \\
\text { not suitable for long-term use }\end{array}$ & Graatsma et al., 2009 [1] \\
\hline Good signal quality during fetal movements & High signal loss during fetal movements & Peters et al., 2001 [2] \\
\hline Signal quality independent of maternal BMI & $\begin{array}{l}\text { Reduced signal quality in overweight/obese } \\
\text { women }\end{array}$ & $\begin{array}{l}\text { Graatsma et al., } 2010 \text { [3]; } \\
\text { Cohen and Hayes-Gill, } 2014 \text { [4] }\end{array}$ \\
\hline $\begin{array}{l}\text { fHR detection during labor more reliable and } \\
\text { accurate than CTG }\end{array}$ & & Cohen et al., 2012 [5] \\
\hline $\begin{array}{l}\text { fHR variations and ST deviation during labor } \\
\text { with accuracy comparable to invasive scalp } \\
\text { fECG }\end{array}$ & fECG morphology not available & $\begin{array}{l}\text { Clifford et al., } 2011 \text { [6]; } \\
\text { Reinhard et al., } 2014 \text { [7] }\end{array}$ \\
\hline Fetal-maternal heart rate confusion less likely & & $\begin{array}{l}\text { Cohen et al., } 2012 \text { [5]; } \\
\text { Stampalija et al., } 2012 \text { [8]; } \\
\text { Reinhard et al., } 2013 \text { [9] }\end{array}$ \\
\hline $\begin{array}{l}\text { Assessment of uterine contractibility by } \\
\text { electrohysterography }\end{array}$ & $\begin{array}{l}\text { Assessment of uterine contractibility by } \\
\text { tocodynamometry }\end{array}$ & $\begin{array}{l}\text { Jacod et al., } 2010 \text { [10]; } \\
\text { Euliano et al., } 2013 \text { [11]; } \\
\text { Euliano et al., } 2016[12]\end{array}$ \\
\hline $\begin{array}{l}\text { Ambulatory monitoring and remote trace } \\
\text { view possible }\end{array}$ & $\begin{array}{l}\text { High signal loss in ambulatory setting; } \\
\text { remote trace view possible }\end{array}$ & $\begin{array}{l}\text { Graatsma et al., } 2009[1] \\
\text { own data }\end{array}$ \\
\hline
\end{tabular}

\section{Disadvantages}

Reduced signal quality at $28-32$ weeks of gestation

Maternal discomfort at electrode site
Reduced signal quality at earlier gestational age

Pressure or belt irritation; fixed position of woman necessary
Graatsma et al., 2009 [1];

Peters et al., 2001 [2];

Piéri et al., 2001 [13]; own data

Graatsma et al., 2009 [1] 


\section{Methods}

\section{Participants}

A prospective observational study was conducted at the Department of Obstetrics and Gynecology, University Hospital Basel, and the Department of Clinical Psychology and Psychotherapy, University of Basel, Switzerland from September 2007 to Septem-

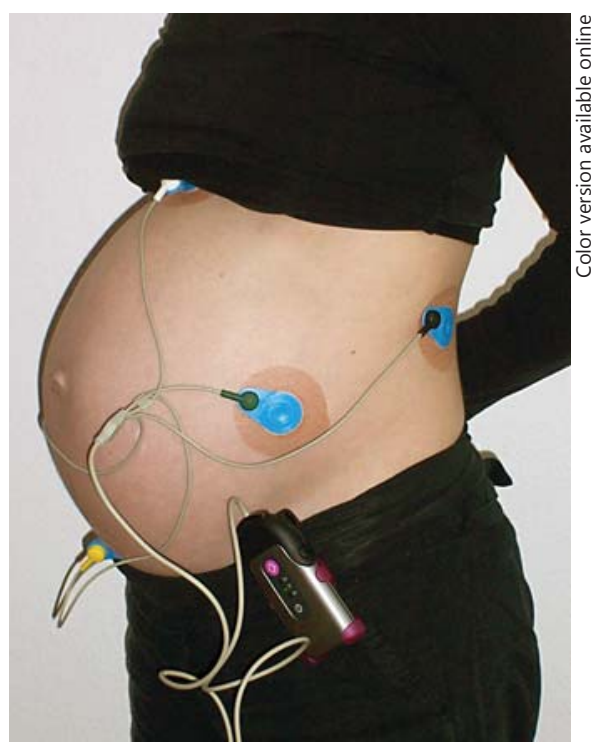

Fig. 1. Pregnant woman wearing the fECG monitor Monica $\mathrm{AN} 24^{\mathrm{TM}}$ at the beginning of the study. Electrode placement was slightly changed according to the manufacturer's suggestions during the trial. ber 2010. The study was approved by the local medical ethics committee (EKBB 74/07).

Women with singleton pregnancies were recruited between 20 and 40 weeks of gestation. Healthy, low-risk pregnant women were asked for a recording at home through flyers in hospitals and via advertisements in local newspapers or the university website. Women with a complicated high-risk pregnancy (e.g., preterm labor, FGR, preeclampsia, placenta previa) were recruited during a hospital stay. The women with preterm labor symptoms were under tocolysis and had no signs of contractions on the previous CTG and during fECG recording. All hospitalized women had regular CTG recordings, which were monitored separately and used for clinical decision-making. Exclusion criteria were known genetic or morphologic abnormalities of the fetus and multiple pregnancies.

\section{ECG Recording and Signal Processing}

After giving written consent, women were assessed with the commercially available and battery-powered Monica AN24 ${ }^{\mathrm{TM}}$ device. The hospitalized women wore the monitor for a posture experiment of $20 \mathrm{~min}$ and could decide thereafter whether to continue the recording during daytime and nighttime. All women at home participated in the posture experiment and in the daytime/ nighttime recording. Two trained investigators attached the electrodes and recording devices, either at the hospital in the clinical group or at the pregnant women's home in the healthy group. Figure 1 shows a picture of the fECG monitor applied to a pregnant woman's abdomen. Details about skin preparation and electrode placement can be found in Graatsma et al. [1]. After analogue filtering, the AN24 ${ }^{\mathrm{TM}}$ samples three ECG channels at $300 \mathrm{~Hz}$. At the beginning of recording and after an automatic impedance check by the $\mathrm{AN} 24^{\mathrm{TM}}$ and a 10 - to 15 -min electrode adaptation period, all included women were asked to stay in the lying position followed by the upright sitting position (or vice versa, counterbalanced for each group) for $10 \mathrm{~min}$ each.

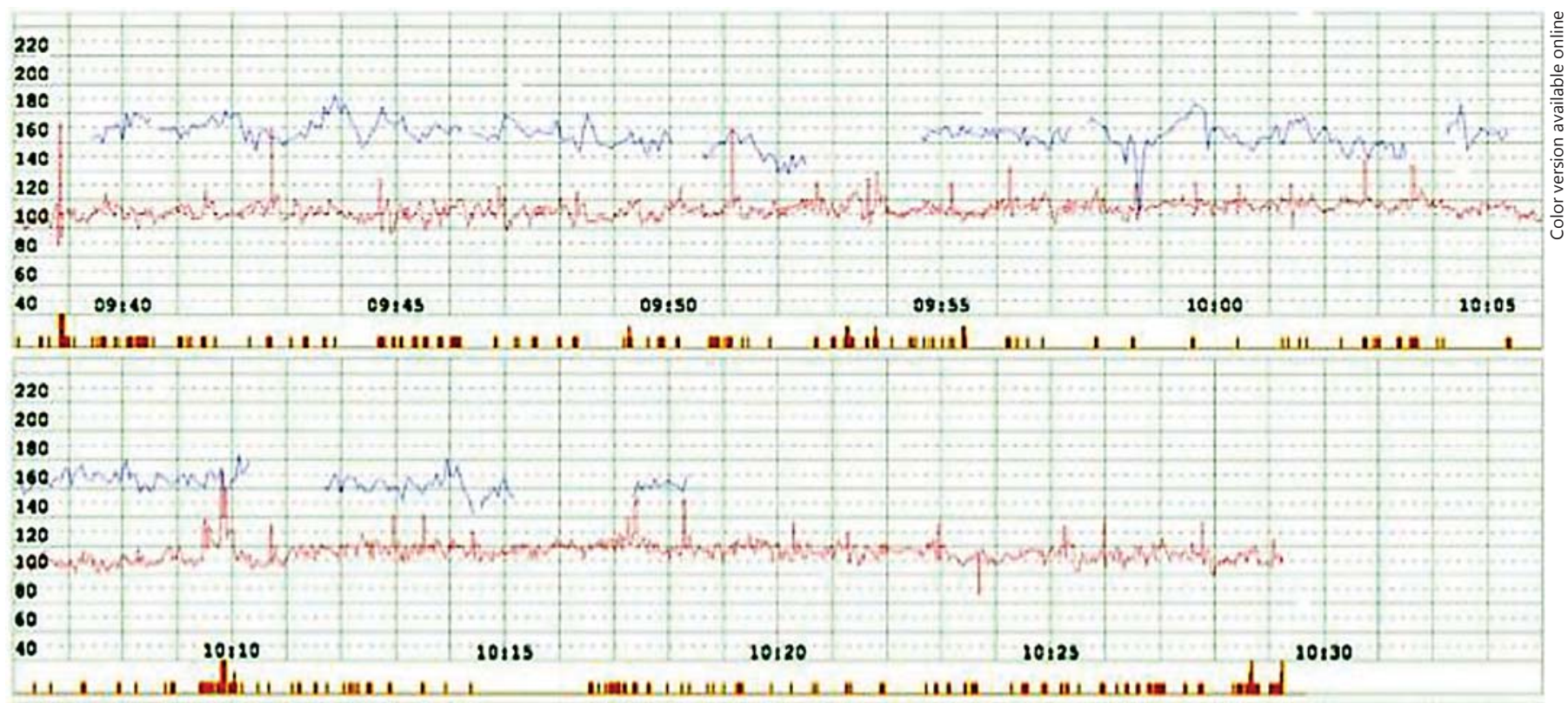

Fig. 2. Display of fetal (blue) and maternal (red) heart rate extracted from recorded ECG signals by the Monica AN24 ${ }^{\mathrm{TM}}$ analysis software.

Abdominal fECG in Ambulatory and Antenatal Bedside Settings
Fetal Diagn Ther 2017;41:283-292

DOI: $10.1159 / 000448946$ 


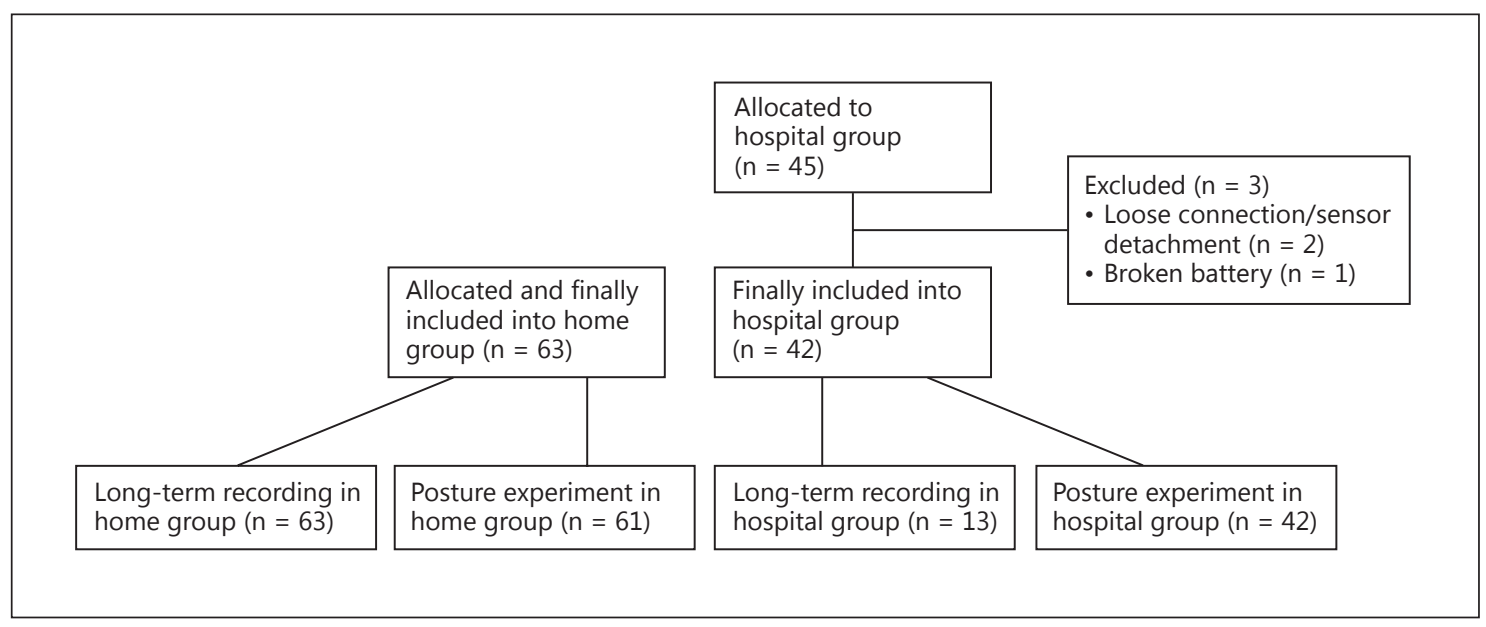

Fig. 3. Flowchart of included and excluded women in the home and hospital groups.

After each recording, data were downloaded from the AN24 ${ }^{\mathrm{TM}}$. Computation of fetal and maternal heart rate was performed automatically offline by the program Monica DevelopmentKit ${ }^{\mathrm{TM}}$ (version 1.3) [13]. Briefly, after detection of maternal ECG and fECG R waves, a third-order polynomial (cube spline) is fitted to the $\mathrm{R}$ waves and is then resampled at $2.1 \mathrm{kHz}$, allowing R-R intervals to be measured with an accuracy of $0.48 \mathrm{~ms}$. The software displays detected beat-to-beat heart rate values and leaves gaps for unclear detection (fig. 2).

\section{Signal Quality Assessment}

The primary outcome variable of signal quality was defined by the success rate (SR) of beat-to-beat fHR detection. The SR is automatically calculated by the signal analysis software of the $\mathrm{AN} 24^{\mathrm{TM}}$ for each minute of recording as the sum of durations of all detected beat-to-beat intervals (in seconds) divided by the entire length of reference period (60 s), expressed as percentage $(0-100 \%)$. The SR was analyzed in relation to time-invariant (trait) and time-variant (state) variables.

\section{Time-Invariant Variables}

Analysis of the SR in relation to time-invariant variables was based on the posture experiment recordings.

\section{Participant Group}

See the 'Participants' section, with specific inclusions reported for the different analyses in the 'Results' section.

\section{Gestational Week}

Weeks of gestation was analyzed as a continuous variable for the main analysis, but was divided into three groups for descriptive purposes (weeks+days): $20+0$ to $27+0,27+1$ to $32+0$, and $32+1$ to $40+0$ weeks of gestation.

\section{Pre-Pregnancy and Current BMI}

The maternal BMI was calculated before pregnancy (attained from a questionnaire) and at the time of recording. The BMI was divided into four classes according to the WHO classification: un- derweight $(<18.50)$, normal (18.50-24.99), overweight (25.0029.99), and obese $(\geq 30.00)$.

\section{Fetal Presentation}

Three different fetal presentations were observed: cephalic, breech, and transverse. The fetal presentation was determined by ultrasound examination up to 7 days before recording. Fetal presentation was not recorded in $14(23 \%)$ healthy women.

\section{Amniotic Fluid Index}

The amniotic fluid was differentiated between normal amniotic fluid index (AFI) and low AFI according to percentiles of gestational age ( $\leq 5$ th percentile). A pathologically high AFI $(\geq 95$ th percentile) was not found in this study. The AFI was measured by ultrasound examination up to 7 days before recording.

\section{Fetal Growth Restriction}

The growth-restricted group was defined by estimated fetal body weight $<10$ th percentile and estimated fetal abdominal circumference $<10$ th percentile for gestational age with normal fetal anatomy. Additionally, the resistance index of the umbilical artery needed to be $\geq 95$ th percentile and the resistance index of the middle cerebral artery $\leq 10$ th percentile for gestational age. The last weight was estimated by ultrasound examination up to 7 days before recording.

\section{Time-Variant Variables}

All time-variant variables were calculated in all women wearing the monitor overnight.

\section{Maternal Physical Activity}

The monitor Monica AN24 ${ }^{\mathrm{TM}}$ has the capability to record and evaluate $3 \mathrm{D}$ accelerometry data. An acceleration value is computed every $2 \mathrm{~s}$ as the sum of the square roots of all axes. Four different physical activity levels are defined by the Monica AN24 ${ }^{\mathrm{TM}}$ software: level 0 - count $<11$; level 2 - count $<100$; level 3 - count $<400$; level 4 - count $\geq 400$. 
Fig. 4. Mean SR of posture manipulation (lying and upright) in three different gestational age groups (low $=20+0$ to $27+0$, medium $=27+1$ to $32+0$, high $=32+1$ to $40+0$ ) in the hospital and home groups.

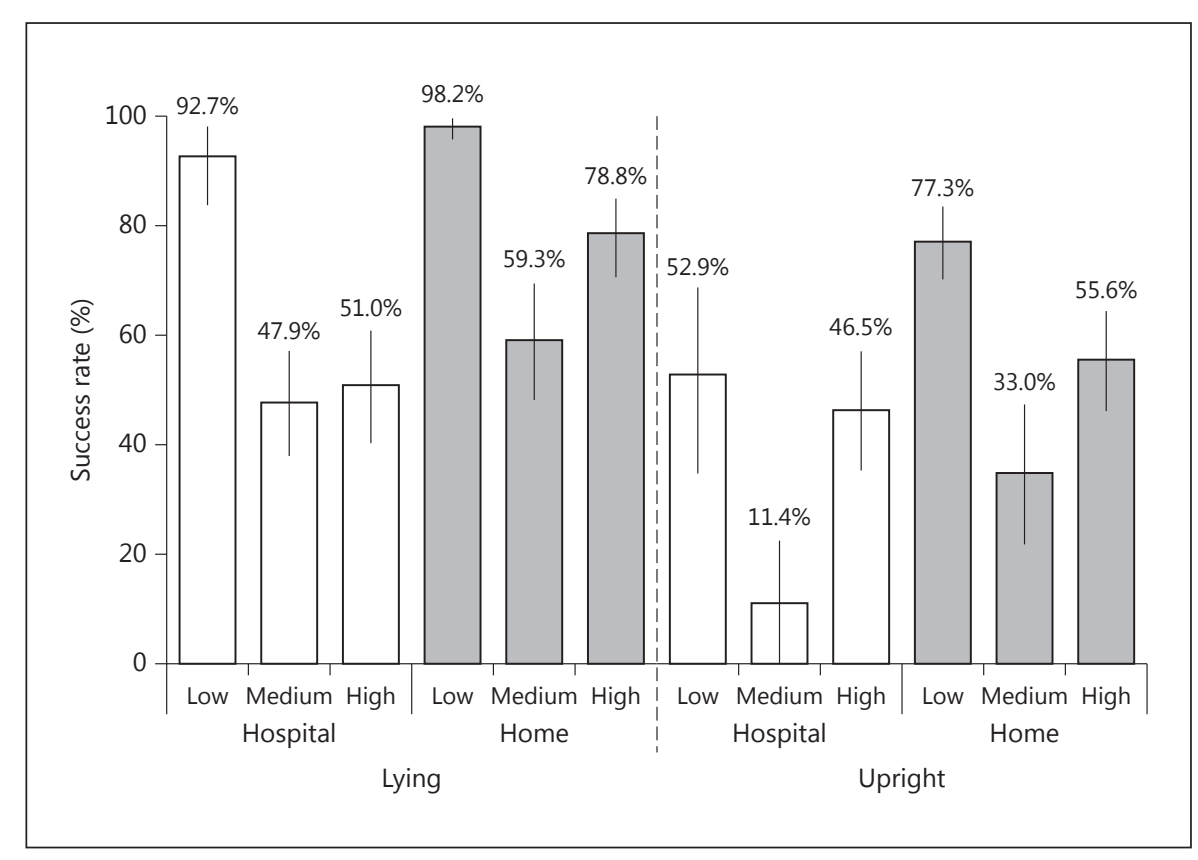

\section{Nighttime versus Daytime}

Nighttime was defined as the period between 11 p.m. and 7 a.m. and daytime as the period between 7 a.m. and 11 p.m.

\section{Circadian Time Intervals}

The data were assigned to six 4-hour intervals: 7:00 p.m. to 10:59 p.m., 11:00 p.m. to 2:59 a.m., 3:00 a.m. to 6:59 a.m., 7:00 a.m. to $10: 59$ a.m., 11:00 a.m. to 2:59 p.m., and 3:00 p.m. to 6:59 p.m.

\section{Statistical Analysis}

SPSS for Windows (version 21) was used for all statistical analyses. Significance was defined as a p value $<0.05$. Statistical tests to compare the two groups with respect to the distribution of various categorical characteristics were all based on Fisher's exact test. The primary outcome variable was the SR of fHR detection, which was arcsine-transformed for inferential statistical testing to better meet model assumptions (normality, homoscedasticity). Since SR was repeatedly measured on the same subjects, linear mixed models [14] were used. Two different mixed models were set up: model A covered the 20-min experimental manipulation period with the full participant sample $(\mathrm{n}=103)$ and tested the impact of posture manipulation and various time-invariant predictors. This model included the factors group (healthy, hospital), posture (lying, upright), and minute of recording (minute 1-20), plus one of the following time-invariant predictors: gestational age $(20+0$ to $27+0$, $27+1$ to $32+0,32+1$ to $40+0$ weeks), current BMI, pre-pregnancy BMI, fetal presentation (cephalic, breech, transverse), AFI (normal, oligohydramnios), and FGR (yes, no). Single time-invariant predictors were included separately rather than together to avoid overfitting. The model contained random coefficients for the intercept, posture manipulation, and time, assuming an autoregressive variance-covariance structure for the residuals. Model B covered the entire recording and included the 76 women who wore the monitor at least overnight. This model included the factor

Abdominal fECG in Ambulatory and Antenatal Bedside Settings group (healthy, hospital), the time-variant predictor of concern, plus the interaction between the two. Time-variant predictors were nighttime versus daytime or time interval. This model contained a random intercept, assuming a compound symmetry variance-covariance structure for the residuals.

\section{Results}

\section{Model A: SR during the Posture Experiment}

Sixty-three healthy and 45 hospitalized pregnant women were recruited. Three women of the hospital group were excluded from analysis because of technical problems (loose connection/sensor detachment and broken battery). Two women of the home group were dropped from analysis because of missing timing information for the posture experiment. Finally, 61 healthy (home) group women versus 42 hospitalized women were included for model A (fig. 3). The maternofetal conditions of hospital stay were: 2 cases of pregnancy-induced hypertension/preeclampsia, 11 cases of FGR, 17 cases of preterm labor with intact membranes, 10 cases of placenta previa/vaginal bleeding, 1 case with gall stones, and 1 case with an upper respiratory infection. Table 2 provides the participant characteristics. The distribution of gestational age groups was significantly different between the hospital and home groups, with $47.6 \%$ of pregnant women at 28-33 weeks of gestation in the hospital group versus $21.7 \%$ in the home group. 
Table 2. Participant characteristics

\begin{tabular}{|c|c|c|c|}
\hline & $\begin{array}{l}\text { Healthy pregnancies } \\
\text { at home }(n=61)\end{array}$ & $\begin{array}{l}\text { High-risk pregnancies } \\
\text { at the hospital }(\mathrm{n}=42)\end{array}$ & $\mathrm{p}$ value \\
\hline Maternal age, years & $31.6 \pm 3.7$ & $31.1 \pm 6.0$ & 0.64 \\
\hline Pre-pregnancy BMI & $22.7(16.7-37.8)$ & $22.9(16.7-33.3)$ & 0.84 \\
\hline Pre-pregnancy BMI subgroups & & & 0.13 \\
\hline$<18.50$ & $1(1.7 \%)$ & $4(9.8 \%)$ & \\
\hline $18.50-24.99$ & $46(79.3 \%)$ & $25(61.0 \%)$ & \\
\hline $25.00-29.99$ & $8(13.8 \%)$ & $10(24.4 \%)$ & \\
\hline$\geq 30.00$ & $3(5.2 \%)$ & $2(4.9 \%)$ & \\
\hline Current BMI & $25.8(20.4-42.8)$ & $27.0(18.7-40.6)$ & 0.19 \\
\hline Current BMI subgroups & & & 0.06 \\
\hline$<18.50$ & $0(0 \%)$ & $0(0 \%)$ & \\
\hline $18.50-24.99$ & $28(49.1 \%)$ & $16(38.1 \%)$ & \\
\hline $25.00-29.99$ & $25(43.9 \%)$ & $16(38.1 \%)$ & \\
\hline$\geq 30.00$ & $4(7.0 \%)$ & $10(23.8 \%)$ & \\
\hline Gestational age, weeks & $30.2(19.6-38.9)$ & $31.3(25.0-36.7)$ & 0.69 \\
\hline Gestational age, weeks+days & & & 0.008 \\
\hline $20+0$ to $27+0$ & $22(36.7 \%)$ & $6(14.3 \%)$ & \\
\hline $27+1$ to $32+0$ & $13(21.7 \%)$ & $20(47.6 \%)$ & \\
\hline $32+1$ to $40+0$ & $25(41.7 \%)$ & $16(38.1 \%)$ & \\
\hline Fetal presentation & & & 0.55 \\
\hline Cephalic & $34(72.3 \%)$ & $31(73.8 \%)$ & \\
\hline Breech & $8(17.0 \%)$ & $9(21.4 \%)$ & \\
\hline Transverse & $5(10.6 \%)$ & $2(4.8 \%)$ & \\
\hline Intrauterine growth restriction & & & $<0.001^{\mathrm{a}}$ \\
\hline Yes & $0(0 \%)$ & $11(26.2 \%)$ & \\
\hline No & $61(100 \%)$ & $31(73.8 \%)$ & \\
\hline AFI & & & $0.025^{\mathrm{a}}$ \\
\hline Normal & $61(100.0 \%)$ & $38(90.5 \%)$ & \\
\hline Oligohydramnios & $0(0 \%)$ & $4(9.5 \%)$ & \\
\hline
\end{tabular}

Figures are given as mean $\pm \mathrm{SD}, \mathrm{n}(\%)$, or median (range).

a Based on Fisher's exact test.

The SR linearly increased over the $20 \mathrm{~min}$ of recording $\left(b=0.452, S E=0.199, F_{1,101}=5.15, p=0.025\right)$. The effects of group and posture manipulation are displayed in figure 4 . The SR was higher in the healthy group (76.1\%) than in the hospital group $(45.5 \% ; \mathrm{b}=0.392, \mathrm{SE}=0.096$, $\left.\mathrm{F}_{1,102}=16.71, \mathrm{p}<0.001\right)$, and also higher in the lying position $(74.6 \%)$ than in the upright position $(47.5 \%$; $\left.\mathrm{b}=0.348, \mathrm{SE}=0.048, \mathrm{~F}_{1,102}=52.16, \mathrm{p}<0.001\right)$. Further, the SR strongly varied among the three gestational age groups, with the $20+0$ to $28+0$ weeks group having the highest values (78.9\%), followed by $32+1$ to $40+0$ weeks group $(49.6 \%)$ and the $28+1$ to $32+0$ weeks group $(29.8 \%$, $\left.\mathrm{F}_{2,102}=13.8, \mathrm{p}<0.001\right)$. There was also a significant interaction between posture manipulation and gestational age $\left(\mathrm{F}_{2,96}=14.1, \mathrm{p}=0.037\right)$. Post-hoc follow-up testing indicated that this was largely due to a particularly lowered SR in in the upright position in the medium gestational age group. Current BMI, pre-pregnancy BMI, and fetal presentation were not related to SR. Estimated means were as follows: pregnancy BMI subgroups - anorectic $44.5 \%$, normal $61.8 \%$, overweight $58.5 \%$, obese $75.6 \%, \mathrm{~F}_{3,99}=$ $0.31, \mathrm{p}=0.82$; current BMI subgroups - normal $67.8 \%$, overweight $59.2 \%$, obese $51.2 \%, \mathrm{~F}_{2,99}=1.16, \mathrm{p}=0.32$; fetal presentations - cephalic $59.2 \%$, breech $69.1 \%$, transverse $80.9 \%, \mathrm{~F}_{2,89}=1.66, \mathrm{p}=0.20$. The predictors AFI and FGR were analyzed only in the hospital group. Low AFI did not differ with respect to SR from normal index (low $12.0 \%$, normal $48.5 \%, \mathrm{~F}_{1,42}=2.89, \mathrm{p}=0.096$ ). Finally, the SR of growth-restricted fetuses did not differ from that of normal fetuses (restricted $48.8 \%$, normal $43.9 \%, \mathrm{~F}_{1,42}=0.13$, $\mathrm{p}=0.72)$. 
Fig. 5. Temporal SR course between 7 p.m. and 6 p.m. in the hospital and home groups.

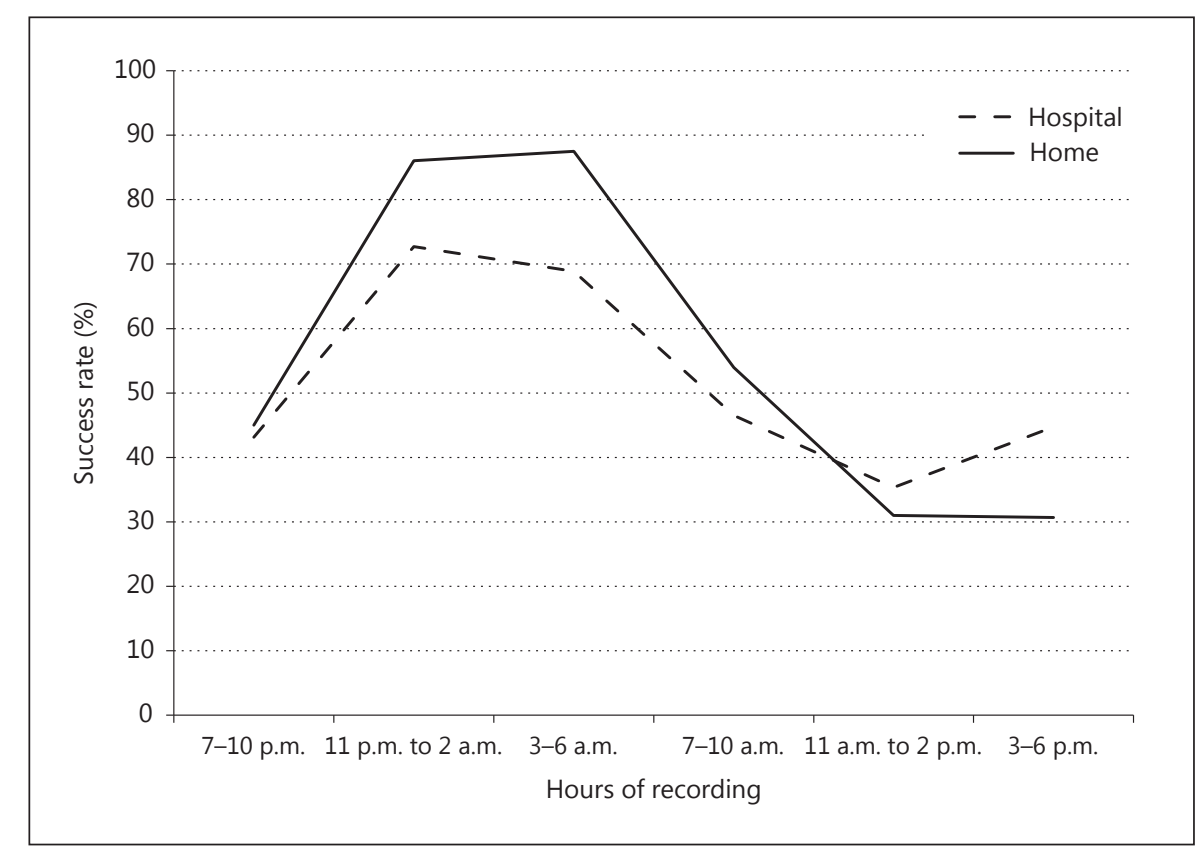

Model B: SR for Long-Term Recording

Following the posture manipulation, the hospital group could decide optionally to prolong the recording. Only 13 women of the hospital group (31\%) agreed. All 63 women of the home group went about their daily lives after posture manipulation and were asked to wear the monitor overnight (fig. 3 , table 2). The median recording time was $18.4 \mathrm{~h}$ with an interquartile range (IQR) of 14.6$22.2 \mathrm{~h}$ (home: median $18.7 \mathrm{~h}$, IQR 15.6-21.8 h; hospital: median $14 \mathrm{~h}$, IQR 10.1-17.9 h). The SR was significantly increased during the nighttime in the hospital group with 71.1 vs. $43.3 \%$ at daytime $\left(\mathrm{F}_{1,87}=19.58, \mathrm{p}<0.001\right)$. The nighttime versus daytime difference was even more pronounced in the home group with 86.8 vs. $40.2 \%\left(\mathrm{~F}_{1,74}=\right.$ $359.91, \mathrm{p}<0.001$ ) (interaction group $\times$ daytime/nighttime, $\left.\mathrm{F}_{1,85}=12.15, \mathrm{p}<0.001\right)$. The low SR for daytime was largely due to the effects of maternal physical activity in the group of healthy women (level 0: 83.2\%; level 1:33.9\%; level 2: 17.0\%; level 3: 8.3\%; $\left.\mathrm{F}_{3,267}=177.32, \mathrm{p}<0.001\right)$. The hospitalized women did not reach a high physical activity level $>1$ and could therefore not be analyzed dependent on their activity level. The temporal course of SR throughout the 24-hour period varied between groups $\left(\mathrm{F}_{5,335}=\right.$ $3.87, \mathrm{p}=0.002)$. Although the highest SR recordings were obtained within the two time intervals 11 p.m. to 2 a.m. and 3 a.m. to 6 a.m. for both groups, averaged values within these intervals were higher in the home group (87\%) compared to the hospital group (69\%) (fig. 5).

Abdominal fECG in Ambulatory and Antenatal Bedside Settings

\section{Discussion}

The current study evaluated variables likely contributing to fHR signal dropout in fECG recording, like home versus hospital setting, maternal BMI, gestational age, fetal presentation and condition, physical activity, posture, and daytime versus nighttime recording. Firstly, results for the full sample based on the highly standardized posture experiment recording (model A) indicate that the SR of fHR detection is not only strongly dependent on maternal posture, but also on the home/hospital setting (fig. 4), even if corrected for various physical characteristics such as BMI, gestational age, and fetal presentation. This observation differs from the results reported by Graatsma et al. [1], who could not find significant differences between home and hospital recordings, with a SR of 95\% (IQR 85-98.4\%) at home and 91.9\% (IQR 77.998.7\%) at the hospital. The lower SR in the hospital group compared to the home group in the present study could be the result of the presence of maternofetal complications. However, occurrence of maternofetal complications such as different fetal presentation or FGR in the hospital group did not significantly influence the SR. The sample size of fetuses with oligohydramnios was low. The SR in fetuses with low AFI appeared to be reduced with 12.0 vs. $48.5 \%$, but this was not significant, most likely due to low sample size. Therefore, the influence of AFI should be evaluated in more detail in future studies.

Fetal Diagn Ther 2017;41:283-292 289 
Factors contributing to the reduced quality of the hospital versus the home setting could also have been gestational age between 28 and 33 weeks of gestation, as previously published $[1,2,13,15]$, due to presence of vernix caseosa, which electrically isolates the fetus. This age group was somewhat overrepresented in the hospital group, which may have contributed to the overall lower SR in the hospital group. However, covariance analysis indicated that the home and hospital groups still differed when taking gestational age into account.

The quality of fECG does not deteriorate with higher maternal BMI as had been shown previously [3], which is getting increasingly important due to the obesity epidemic in women of reproductive age. The relative accuracy of fECG recording in obese women is a major advantage over the Doppler ultrasound recordings using CTG, with performance of fHR recordings degrading directly dependent on maternal BMI [4].

Interestingly, according to the manufacturer's recommendations of 10-15 min rest after electrode application and device calibration before starting the recording, we still detected a linear improvement of the recording during the subsequent standardized 20 -min posture experiment. This suggests that the SR during the posture experiment could have been somewhat higher with longer resting time before the start of recording.

Analyses based on long-term recordings (time-variant analyses, model B) indicate that a clear limitation of abdominal fECG recording is fHR signal dropout due to maternal muscle activity. This is demonstrated by the poor SR dependent on maternal activity level in the home group (SR of $17 \%$ in level 2 and $8.3 \%$ in level 3). The observed higher signal dropout with the upright compared to the lying position both in the posture experiment and the long-term data is likely also due to higher isometric abdominal muscle contractions. Such skeletal muscle artefacts result in high-frequency noise in the ECG signal of $10-500 \mathrm{~Hz}$, which appears to make reliable fetal $\mathrm{R}$ wave detection difficult or even impossible with current technology. Long-term recordings also clearly indicate that the quality of the fECG signal is best during overnight recordings when the woman rests supine and abdominal muscle activity is minimized. This applies to recordings at home (SR $86.8 \%$ for nighttime vs. $40.2 \%$ for daytime) and in the hospital (71.1\% for nighttime vs. $43.3 \%$ for daytime). But even in the overnight recordings, our SR was lower than that previously reported by Graatsma et al. [1], who used the same ECG monitor (Monica AN24 ${ }^{\mathrm{TM}}$ ). In addition, nighttime recordings confirm the lower in-hospital SR found in the standardized posture experiment. During our data acquisition, we were conscious of the relatively low SR compared to Graatsma et al.'s data. However, even under constant supervision, training and support from Monica Healthcare Ltd., checkup of the two employed Monica AN24 ${ }^{\mathrm{TM}}$ devices by the company, and variation in abdominal electrode placement, the SR did not show systematic improvements.

We think that environmental factors might have had a particular influence on the low in-hospital SR. Electrical power lines and devices in the hospital environment are known to cause electromagnetic interference, making it difficult to detect faint fECG signals. This may apply both to the standardized posture experiment and the unsupervised long-term recordings. In addition, during nighttime recordings the clinical group might have been more anxious, uncomfortable, and sleepless due to their novel situation and interruptions by other hospitalized women and staff. Such factors could be measured and systematically taken into account in future studies. In any case, such differences reflect clinical reality, and thus the present results can probably be considered ecologically valid. Since our study, fECG recordings might have been somewhat improved by the use of newer software (Monica DevelopmentKit ${ }^{\mathrm{TM}}$ version 1.9 instead of version 1.3) and a slightly changed recommended electrode placement. Only $31 \%$ of all pregnant women at the hospital were willing to continue the fECG recordings if asked after the 20-min posture recording. This indicates that acceptance of fECG recording with 5 electrodes from the abdomen was low for longer periods within a voluntary experimental setting. Many women participating in the overnight recording reported some discomfort such as itching of the electrode site, skin irritation, or hindered night rest. Generally, women hospitalized for pregnancy complications are likely more reluctant to participate in any type of experimental procedure compared to healthy pregnant women. Acceptance is likely much higher in medically indicated conditions where long-term fHR recording may provide important diagnostic information.

Our study does not provide information about restrictions of fetal assessment in the preterm labor group because it did not investigate the signal quality under antenatal uterine contractions. Our preterm women were in part under tocolytic medication and had no uterine contractions during recordings. Statements about influences of tocolytics or other medications, alcohol or nicotine consumption cannot be made by this study.

The average SR of $40.2 \%$ for the home and $43.3 \%$ for the hospital group during daytime might not be high enough for fHRV analysis, especially for short-term vari- 
ability indices, which are currently used in the monitoring of FGR [16]. Dawes et al. [17] defined a recording as sufficient for short- and long-term variability measurement if the recording had a high recording quality of $\geq 60 \%$. First attempts to investigate fHRV by short-term variation using abdominal fECG were recently conducted in overnight recordings [18-20]. A new signal-processing technique, phase-rectified signal averaging, was used to minimize noise by averaging signals. It provides separate quantification of acceleration- and deceleration-related heart rate modulations $[18,21,22]$. Only 3 of $30(10.0 \%)$ fECG recordings of growth-restricted fetuses and 5 of 90 (5.6\%) normal estimated fetuses had to be excluded due to poor signal quality in overnight recordings [18]. Using high-resolution fECG data, the new analysis technique could better discriminate between normal fetuses and fetuses who were small for gestational age than conventional short-term variation measures. Another study investigated the applicability of spectral analysis in abdominal fECG recording [23]. Forty women were studied longitudinally throughout pregnancy with an abdominal 8-channel ECG monitor (ECG prototype called NEMO) with a sampling rate of $1,000 \mathrm{~Hz}$ and a recording duration of $45 \mathrm{~min}$ at daytime. In these short-term recordings, only $3 \%$ of the abdominally retrieved data were usable for spectral analysis due to poor signal quality after artefact correction (only 64 -second segments with $<5 \%$ of artefact correction were included). Therefore, we consider overnight long-term recordings the applicable way of antenatal surveillance of high-risk pregnancies for assessment of clinically relevant fHRV indices. New research perspectives for fECG might be to investigate maternal and fetal heart rate variability simultaneously and thereby to study influences of intrinsic and extrinsic stimuli on fHRV and the psychophysiological linkage (coherence) between mother and fetus [24]. The accuracy of fHRV measurements based on fECG compared to the gold standard, CTG, and the quality of antenatal fECG morphology especially in high-risk pregnancies needs to be further investigated.

\section{Conclusion}

The SR of fHR detection is strongly dependent on the home/hospital setting and different predictors such as time of recording, activity levels, and maternal posture. The quality of the fECG signal is best during overnight recordings in the home and hospital setting, when the woman rests supine and abdominal muscle activity is minimized. Factors contributing to reduced quality are gestational age of 28-33 weeks. The technique can be reasonable for close long-term surveillance in a clinical antenatal high-risk group or in obese women who typically are difficult to assess with CTG antenatally. Particularly when women are monitored at home, precautions should be taken to minimize physical activity and posture shifts during periods of interest to assure sufficient data quality.

\section{Acknowledgments}

The authors would like to thank Monica Healthcare Ltd. for continuous technical support during this study. Dr. Terence Martin from Monica Healthcare Ltd. commented on an early version of the manuscript. Special thanks go to all families who participated in the study and the kindergartens, day nurseries, midwives, and pediatricians who supported our study by encouraging suitable healthy women to participate.

\section{Disclosure Statement}

This work is part of the National Centre of Competence in Research (NCCR) Swiss Etiological Study of Adjustment and Mental Health (SESAM). The Swiss National Science Foundation (SNF) (project no. 51A240-104890), the University of Basel, the F. Hoffmann-La Roche Corporation, and the Swiss Scientific Society provided core support in addition to the NCCR SESAM. The authors declare that there are no further financial or personal relationships with other people or organizations that could inappropriately have influenced the work reported or the conclusions, implications, or opinions stated.

\section{References}

Abdominal fECG in Ambulatory and

Antenatal Bedside Settings
1 Graatsma EM, Jacod BC, van Egmond LAJ, Mulder EJH, Visser GHA: Fetal electrocardiography: feasibility of long-term fetal heart rate recordings. BJOG 2009;116:334-337.

2 Peters M, Crowe J, Piéri JF, Quartero HWP, Hayes-Gill B, James D, Stinstra J, Shakespeare S: Monitoring the fetal heart non-invasively: a review of methods. J Perinat Med 2001;29: 408-416.
3 Graatsma EM, Miller J, Mulder EJ, Harman C, Baschat AA, Visser GH: Maternal body mass index does not affect performance of fetal electrocardiography. Am J Perinatol 2010;27: 573-577.

4 Cohen WR, Hayes-Gill B: Influence of maternal body mass index on accuracy and reliability of external fetal monitoring techniques. Acta Obstet Gynecol Scand 2014;93:590-595. 
5 Cohen WR, Ommani S, Hassan S, Mirza FG, Solomon M, Brown R, Schifrin BS, Himsworth JM, Hayes-Gill BR: Accuracy and reliability of fetal heart rate monitoring using maternal abdominal surface electrodes. Acta Obstet Gynecol Scand 2012;91:1306-1313.

6 Clifford G, Sameni R, Ward J, Robinson J, Wolfberg AJ: Clinically accurate fetal ECG parameters acquired from maternal abdominal sensors. Am J Obstet Gynecol 2011;205: 47.e1-e5.

7 Reinhard J, Hayes-Gill B, Yuan J, Schiermeier S, Louwen F: Intrapartum ST segment analyses (STAN) using simultaneous invasive and non-invasive fetal electrocardiography: a report of 6 cases. Z Geburtshilfe Neonatol 2014; 218:122-127.

8 Stampalija T, Signaroldi M, Mastroianni C, Rosti E, Signorelli V, Casati D, Ferrazzi EM: Fetal and maternal heart rate confusion during intra-partum monitoring: comparison of trans-abdominal fetal electrocardiogram and Doppler telemetry. J Matern Fetal Neonatal Med 2012;25:1517-1520.

9 Reinhard J, Hayes-Gill BR, Schiermeier S, Hatzmann H, Heinrich TM, Louwen F: Intrapartum heart rate ambiguity: a comparison of cardiotocogram and abdominal fetal electrocardiogram with maternal electrocardiogram. Gynecol Obstet Invest 2013;75:101108.

10 Jacod BC, Graatsma EM, van Hagen E, Visser GHA: A validation of electrohysterography for uterine activity monitoring during labour. J Matern Fetal Neonatal Med 2010;23:17-22.

11 Euliano TY, Nguyen MT, Darmanjian S, McGorray SP, Euliano N, Onkala A, Gregg AR:
Monitoring uterine activity during labor: a comparison of 3 methods. Am J Obstet Gynecol 2013;208:66.e1-e6.

12 Euliano TY, Nguyen MT, Darmanjian S, Busowski JD, Euliano N, Gregg AR: Monitoring uterine activity during labor: clinician interpretation of electrohysterography versus intrauterine pressure catheter and tocodynamometry. Am J Perinatol 2016;33:831-838.

13 Piéri JF, Crowe JA, Hayes-Gill BR, Spencer CJ, Bhogal K, James DK: Compact long-term recorder for the transabdominal foetal and maternal electrocardiogram. Med Biol Eng Comput 2001;39:118-125.

14 Singer JD, Willett JB: Applied Longitudinal Data Analysis. Modeling Change and Event Occurrence. New York, Oxford University Press, 2003.

15 Oostendorp TF, van Oosterom A, Jongsma HW: The effect of changes in the conductive medium on the fetal ECG throughout gestation. Clin Phys Physiol Meas 1989;10(suppl B):11-20.

16 Pardey J, Moulden M, Redman CWG: A computer system for the numerical analysis of nonstress tests. Am J Obstet Gynecol 2002; 186:1095-1103.

17 Dawes GS, Houghton CR, Redman CW, Visser GH: Pattern of the normal human fetal heart rate. Br J Obstet Gynaecol 1982;89:276284.

18 Graatsma EM, Mulder EJH, Vasak B, Lobmaier SM, Pildner von Steinburg S, Schneider KTM, Schmidt G, Visser GHA: Average acceleration and deceleration capacity of fetal heart rate in normal pregnancy and in pregnancies complicated by fetal growth restric- tion. J Matern Fetal Neonatal Med 2012;25: 2517-2522.

19 Stampalija T, Casati D, Montico M, Sassi R, Rivolta MW, Maggi V, Bauer A, Ferrazzi E: Parameters influence on acceleration and deceleration capacity based on trans-abdominal ECG in early fetal growth restriction at different gestational age epochs. Eur J Obstet Gynecol Reprod Biol 2015;188:104-112.

20 Hofmeyr F, Groenewald CA, Nel DG, Myers MM, Fifer WP, Signore C, Hankins GD V, Odendaal HJ: Fetal heart rate patterns at 20 to 24 weeks gestation as recorded by fetal electrocardiography. J Matern Fetal Neonatal Med 2014;27:714-718.

21 Huhn EA, Lobmaier S, Fischer T, Schneider R, Bauer A, Schneider KT, Schmidt G: New computerized fetal heart rate analysis for surveillance of intrauterine growth restriction. Prenat Diagn 2011;31:509-514.

22 Lobmaier SM, Huhn EA, Pildner von Steinburg S, Müller A, Schuster T, Ortiz JU, Schmidt G, Schneider KT: Phase-rectified signal averaging as a new method for surveillance of growth restricted fetuses. J Matern Fetal Neonatal Med 2012;25:2523-2528.

23 Van Laar JOEH, Warmerdam GJJ, Verdurmen KMJ, Vullings R, Peters CHL, Houterman S, Wijn PFF, Andriessen P, van Pul C, Guid Oei S: Fetal heart rate variability during pregnancy, obtained from non-invasive electrocardiogram recordings. Acta Obstet Gynecol Scand 2014;93:93-101.

24 Gates KM, Gatzke-Kopp LM, Sandsten M, Blandon AY: Estimating time-varying RSA to examine psychophysiological linkage of marital dyads. Psychophysiology 2015;52:1059-1065. 